\title{
White Patch on the Fore-Flipper of Common Minke Whale, as a Potential Morphological Index to Identify Stocks
}

\author{
Gen Nakamura ${ }^{*}$, Ichiro Kadowaki ${ }^{1}$, Shouka Nagatsuka1, Ryotaro Hayashi', \\ Naohisa Kanda', Mutsuo Goto ${ }^{3}$, Luis A. Pastene ${ }^{3}$, Hidehiro Kato ${ }^{1}$ \\ ${ }^{1}$ Laboratory of Cetacean Biology, Tokyo University of Marine Science and Technology, Tokyo, Japan \\ 2Japan NUS Company Ltd., Tokyo, Japan \\ ${ }^{3}$ Institute of Cetacean Research, Tokyo, Japan \\ Email: "gnakam1@kaiyodai.ac.jp
}

Received 14 January 2016; accepted 10 April 2016; published 13 April 2016

Copyright (C) 2016 by authors and Scientific Research Publishing Inc.

This work is licensed under the Creative Commons Attribution International License (CC BY). http://creativecommons.org/licenses/by/4.0/

(c) $\underset{\mathrm{EY}}{\mathrm{E}}$ Open Access

\begin{abstract}
Past studies have indicated that there are two different stocks ( $\mathrm{J}$ and $\mathrm{O}$ stocks) in the North Pacific common minke whale Balaenoptera acutorostrata population. These two stocks differ from each other in certain characteristics, such as body size, conception dates, and genetics. However, till date, few studies have investigated differences in the external body appearance between the two stocks. Therefore, in the present study, we focused on the unique white patch on the flipper of this whale species to elucidate inter-stock differences. We used the animals collected from JARPNII research during 2012 and 2013; stock information was determined by microsatellite DNA analysis $(n=220)$. We focused on the morphological differences in the size and pattern of the white patch on the flipper of each whale. The length of the white patch along the anterior (ventral) margin of the flipper tends to be proportionally larger in 0 stock. The pattern of the boundary area of the white patch named as the "Grayish Accessary Layer (GAL)" was remarkably different between stocks. Within animals with "no GAL" type, $\mathbf{9 4 \%}$ were J stock. Conversely, of animals with GAL expanding over the half the flipper width, $96 \%$ were 0 stock. We concluded from our study that there were clear morphological differences in the flipper color pattern between $\mathrm{J}$ and $\mathrm{O}$ stocks, which enable to apply widely to other individuals.
\end{abstract}

\section{Keywords}

Common Minke Whale, White Patch, External Morphology, Stock

${ }^{*}$ Corresponding author.

How to cite this paper: Nakamura, G., Kadowaki, I., Nagatsuka, S., Hayashi, R., Kanda, N., Goto, M., Pastene, L.A. and Kato, H. (2016) White Patch on the Fore-Flipper of Common Minke Whale, as a Potential Morphological Index to Identify Stocks. Journal of Biomaterials and Nanobiotechnology, 7, 116-122. http://dx.doi.org/10.4236/ojas.2016.62014 


\section{Introduction}

The common minke whale Balaenoptera acutorostrata is the smallest species of the Balaenoptera whales. In the northern hemisphere, there are two distinguishable subspecies, one each in the North Pacific (B. a. scammo$n i)$ and North Atlantic (B. a. acutorostrata). In the southern hemisphere, there is another population, generally called as "dwarf" minke whales. They may provisionally be regarded as a racesubspecies of common minke whale, but their taxonomical status is not fixed yet due to the scanty data [1]. The notable feature of the common minke is the white patch in their flipper which is the unique character of this species. The size and the shape of the white patch differ between subspecies. Therefore, the white patch on the flipper may serve as a useful distinguishing characteristic for taxonomy classification purposes [2] [3].

Historically, this species have been regarded as an important resource worldwide. For the proper management of wild resources, stock-based management is important as well as species-based management. For the North Pacific common minke whales, the attempts to clarify the stock structure began in the 1950s. In the 1980s, it was suggested that there were two minke whale stocks in seas around Japan [4] comprising $\mathbf{J}$ stock which is mainly distributed from the Sea of Japan to the East China Sea, and O stock which is distributed from the Okhotsk Sea to the Western North Pacific. The two whale stocks share the same feeding ground and they are mixed in the Okhotsk Sea and Japanese coasts [5] [6].

Various aspects of the stock structure of the North Pacific minke whale population have been studied in-depth, including work on genetics [7]-[9], conception dates [10], and cookie cutter shark-induced scar marks [11]. Morphological studies comparing body length, body proportions, color patterns of the baleen plates, flippers and tail flukes have also been conducted [4] [10] [12] [13].

Among North Pacific common minke whales, it has been reported that the area ratio of the white patch on the flipper differs between J and O stocks [13]. Kanda et al. (2010) [13] suggested that the white patch length in proportion to the overall flipper length may differ; however, there was still no clear indicator to identify the two stocks. Therefore, we focused on the white patch of the flipper of the North Pacific minke whale in more detail. The aim of this study was to clarify the morphological differences between stocks so that a morphological index could be developed which would help to correctly identify whale stocks from their appearance.

\section{Materials and Methods}

\subsection{Materials}

The samples were collected during offshore and coastal (Sanriku and Kushiro) research of the Japanese Whale Research Program under Special Permit in the Western North Pacific-Phase II (commonly known as JARPNII) (Figure 1). The survey was conducted in accordance with the Article VIII of the International Convention for the

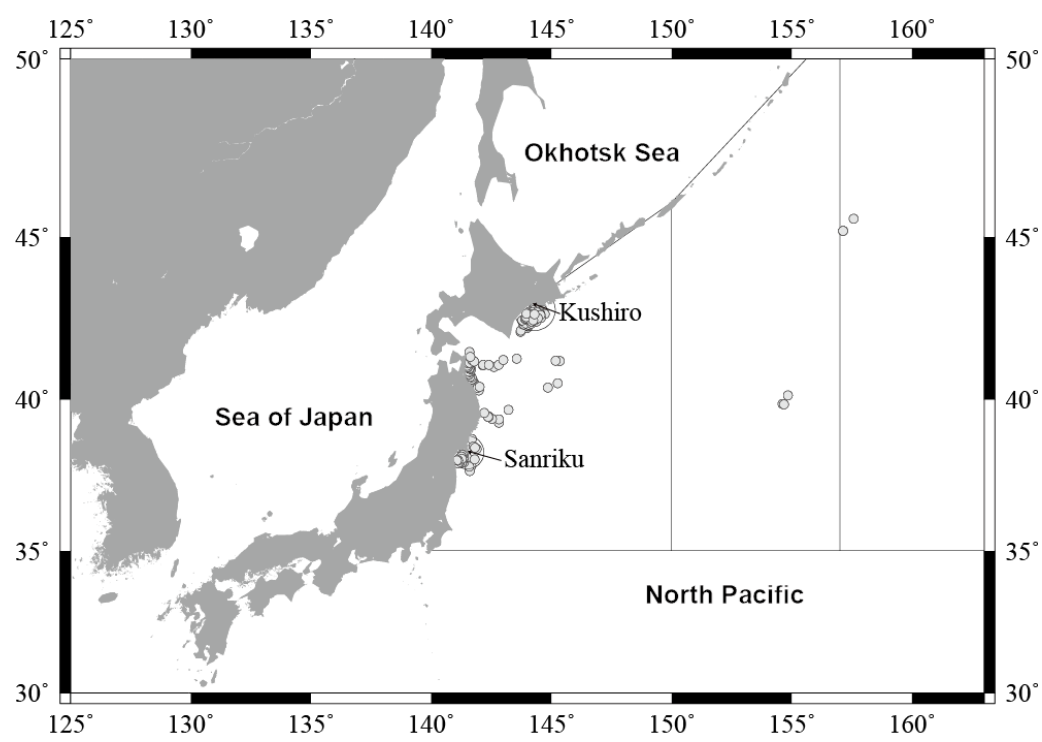

Figure 1. Research area (surrounded by line) and sampling location (circle). 
Regulation of Whaling. The animals were identified and classified into either $\mathrm{J}$ or $\mathrm{O}$ stock using genetic analyses along with a combination of microsatellite and Bayesian clustering analyses following the methods of Kanda et al. (2009) [9]. We used the data collected from 2012 and 2013, because flipper measurements were taken in these two seasons. We conducted research on 220 animals (138 males, body length: $3.82-8.16 \mathrm{~m} ; 82$ females, body length: $4.06-8.68 \mathrm{~m}$ ), which were assigned into the appropriate stock based on their high membership probability $(>90 \%)$.

\subsection{Measurement and Classification of the Flipper Types}

In this study, we focused on the proportional size and boundary area pattern of the white patch on the flipper of each whale. We measured the following three flipper characteristics after the flipper was dissected from the ance from the tip of the flipper to the distal end of the white patch (Point A), and the tip to mesial end of the white patch (Point B) (Figure 2). These points were measured to millimeter scale accuracy using measuring tape or a stainless steel caliper. The boundary area pattern of the white patch named as "Grayish Accessary Layer (GAL)" were classified into groups, based on the criteria described below (Figure 3).

No GAL type: Animals with no grayish band near the boundary;

Type 1: With small GAL not fused to the other side;

Type 2: GAL is connected to the other side;

Type 3: GAL expands over the half-line of the flipper width.

\subsection{Statistical Analysis}

In the process of the statistical analysis, Mann-Whitney $U$-test was adopted to compare the proportional size of the white patch on the flipper and Pearson's chi-square test was adopted to compare the frequency of GAL types between two stocks. Each analysis were operated using the statistic software R 2.13.0.

\section{Results}

\subsection{Proportional Size of the White Patch on the Flipper}

The proportional length of Point A (distance from the tip of the flipper to the distal end of the white patch) to the

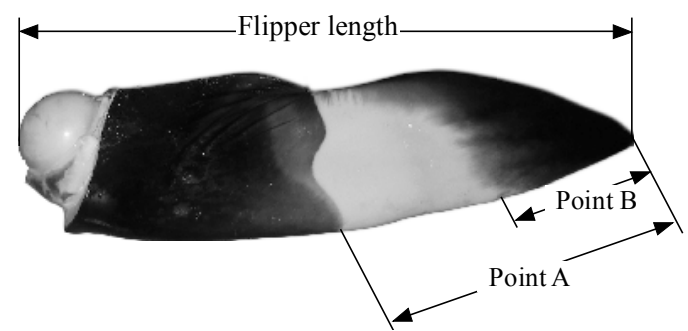

Figure 2. Measurement points on the flipper.
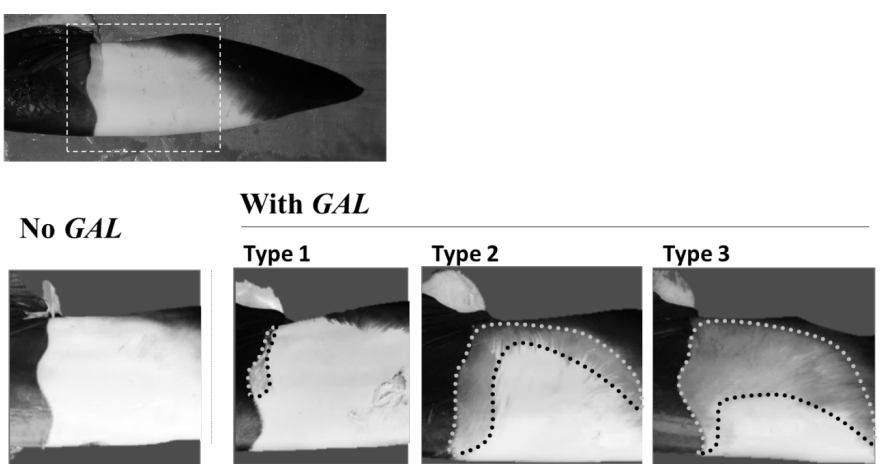

Figure 3. Basis for the classification based on the GAL types (GAL: surrounded by dotted line). 
flipper length was $61.3 \% \pm 4.7 \%(n=47)$ and $63.8 \% \pm 4.5 \%(n=168)$ for $\mathrm{J}$ and O stocks, respectively. Although the values overlapped, the distal end of the white patch tends to be relatively far from the tip of flipper in O stocks compared with that in J stock whales (Mann-Whitney $U$-test, $p<0.01$ ) (Figure 4(a)).

The proportional length of Point B (distance from the tip of the flipper to the mesial end of white patch) to the flipper length was $29.7 \pm 4.5 \%(n=49)$ and $30.3 \pm 4.7 \%(n=169)$ for $\mathrm{J}$ and O stocks, respectively. The values are quite similar between the two stocks, and therefore, no statistical differences were observed in this characteristic (Mann-Whitney $U$-test, $\mathrm{p}>0.05$ ) (Figure 4(b)).

\subsection{Classification by GAL Type}

The relationship between the type of stock and pattern of the boundary in the white patch (GAL) of individual whales was studied. More than $80 \%$ whales were categorized as Types $1(n=32)$ and $2(n=143)$ comprised both stocks. However, 94\% of the "no GAL" type $(n=16)$ whales comprised J stock, and $96 \%$ of Type 3 ( $n=$ 27) whales comprised $O$ stock (Table 1, Figure 5). The frequency of the $J$ and $O$ stocks was distinct among GAL types (Pearson's chi-square test, $\mathrm{p}<0.01$ ).

\section{Discussion}

The present study reported that the pattern of the white patch correlate well with genetics and this character would be help to identify stocks from external appearance. Previous studies mentioned the possibility that the pattern of the white patch on the flipper differs in these whales within as well as between particular oceans/seas, thus suggesting the possibility of inter-stock differences [3] [10] [14]. As indicated by these studies, definite morphological differences in the white patch of the flipper were observed between stocks.

a) Point $\mathrm{A}$

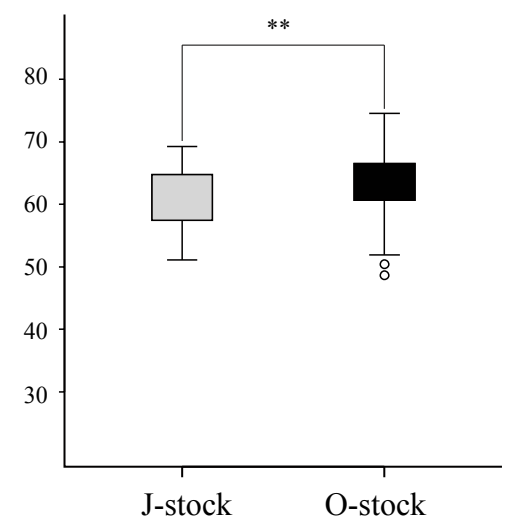

b) Point B

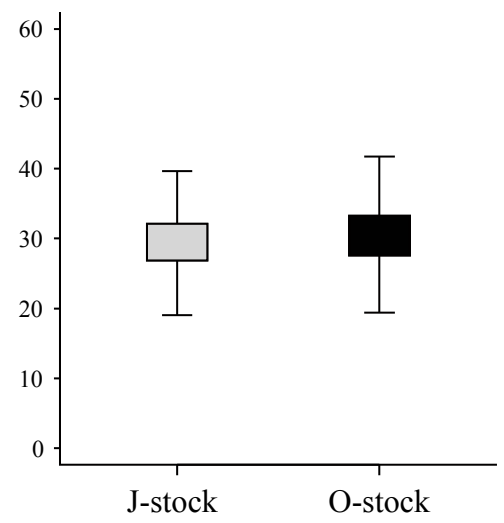

Figure 4. Proportional size of white patch in the flipper. a) The proportional length of Point A (the distance from the tip of the flipper to the distal end of white patch) to the flipper length; b) The proportional length of Point B (the distance from the tip of the flipper to the mesial end of white patch) to the flipper length. In Point A, proportional length was larger wain O stocks compared to J stock whales (Mann-Whitney U-test, $\mathrm{p}<0.01$ ).

Table 1. Number and proportion of each GAL types between $\mathrm{J}$ and $\mathrm{O}$ stocks.

\begin{tabular}{cccccc} 
& & & With GAL & Total \\
\cline { 3 - 4 } No GAL & & Type 1 & Type 2 & Type 3 & \\
J stock & 15 & 7 & 27 & $(3.7 \%)$ & 50 \\
& $(93.8 \%)$ & $(21.9 \%)$ & $(18.9 \%)$ & 26 & 168 \\
O stock & 1 & 25 & 116 & $(96.3 \%)$ & 218 \\
\hline
\end{tabular}




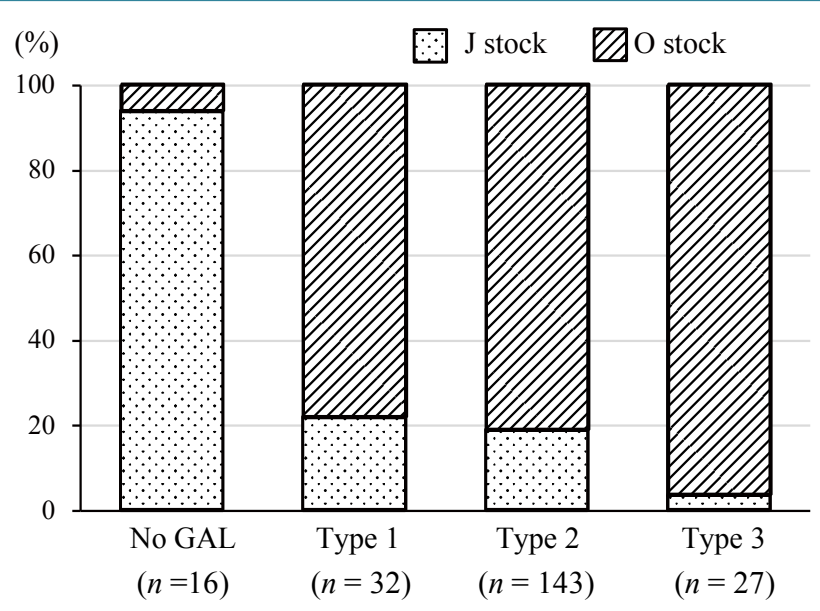

Figure 5. The relationships between stock and the pattern of the boundary in white patch (GAL).

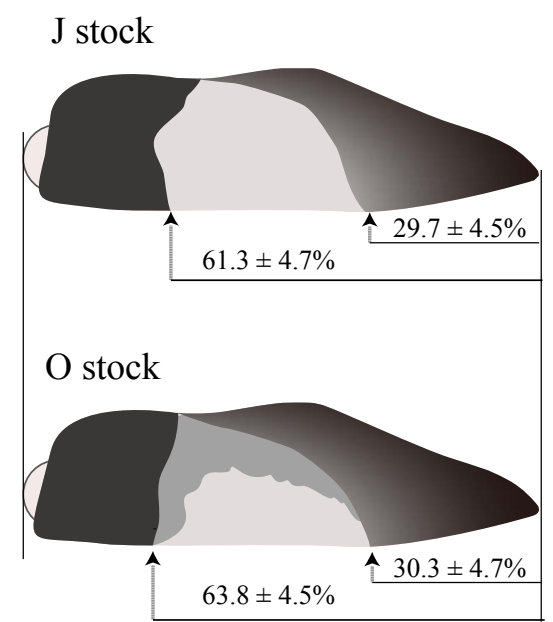

Figure 6. Typical diagram of the white patch of the flipper in $\mathrm{J}$ and $\mathrm{O}$ stocks.

The length of Point B was not significantly different between stocks, whereas the length of Point A was proportionally larger in $\mathrm{O}$ stock than in J stocks. This meant that the length of white patch along the anterior (ventral) margin of the flipper tends to be proportionally larger in O stocks. Statistical analyses also support these results. However, the range of the proportion are overlapped between stocks, and therefore, it would be difficult to classify whales into either stock with $100 \%$ accuracy based only on this characteristic. The GAL pattern was the clearest characteristic for stock identification. We were able to define separate stocks with high probability $(>90 \%)$ when the target animal was "no GAL" type or "Type 3". These findings may help in the future development of a useful index to identify different North Pacific common minke whale stocks (Figure 6).

At present, the stock of each animals are identified by the combination method of microsatellite and Bayesian clustering analyses following the methods of Kanda et al. (2009) [9], as mentioned above. In this method, the database is required to identify stocks from the animals collected by stranding or biopsy sampling. However, if the flipper could be observed and its GAL type was "no GAL" or "Type 3", the stock is identified with high accuracy.

On the other hand, more than $80 \%$ of observed animals were classified into Type $1(15 \%, n=32)$ or $2(66 \%$, $n=143$ ), and these were from both J and O stocks. For such animals, by combining other external characteristics, such as cookie cutter shark-induced scar marks and unsolved indicators that include body color pattern, the accuracy of whale stock identification in the ocean will be greatly improved and also would be able to clarify distribution areas of each stocks. 


\section{Conclusion}

This study declared that white patch of the common minke whales was morphologically different between two stocks. The size of the white patch area relative to flipper area was not significantly different, however, the boundary area pattern of the white patch named as "Grayish Accessary Layer (GAL)" correlated highly with genetics. Using these characters, for the animal having typical character, it would be able to distinguish stocks only from its appearance.

\section{Acknowledgements}

We thank the former leaders of the JARPNII Survey, Genta Yasunaga and Takeharu Bando of the Institute of Cetacean Research, Toshiya Kishiro and Hideyoshi Yoshida of the National Research Institute of Far Seas Fisheries, Representative Director Yoshiichi Shimomichi, former Exploration Chief Shigeo Tabata, Takahiro Hara and the staff of the Association for Community-Based Whaling, the staff of Kyodo Senpaku Company who is the main proponent of offshore surveys. We also thank to the anonymous reviewers and the members of the Laboratory of Cetacean Biology, Tokyo University of Marine Science and Technology. The authors would like to thank Enago (www.enago.jp) for the English language review.

\section{References}

[1] Rice, D.W. (1998) Marine Mammals of the World. Systematics and Distribution, Society for Marine Mammalogy Special Publication 4.

[2] Kato, H. and Fujise, Y. (2000) Dwarf Minke Whales; Morphology, Growth and Life History with Some Analyses on Morphometric Variation among the Different Forms and Regions. Paper SC/52/OS3 Presented to the Scientific Committee, June 2000, 30 p. (Unpublished)

[3] Nakamura, G., Kadowaki, I., Nagatsuka, S., Fujise, Y., Kishiro, T. and Kato, H. (2014) Variation in a Color Pattern of White Patch on the Flippers of North Pacific Common Minke Whales: Potential Application for Their Interoceanic Difference. La Mer, 52, 31-47.

[4] Ohsumi, S. (1983) Minke Whales in the Coastal Waters of Japan in 1981, with Special Reference to Their Stock Boundary. Reports of the International Whaling Commission, 33, 365-371.

[5] Ohsumi, S. (1977) Minke Whales in the Coastal Waters of Japan. Reports of the International Whaling Commission, 27, 164-166.

[6] Hatanaka, H. and Miyashita, T. (1997) On the Feeding Migration of Okhotsk Sea. West Pacific Stock of Minke Whales, Estimates Based on Length Composition Data. Reports of the International Whaling Commission, 47, 557564.

[7] Wada, S. (1991) Genetic Structure of Okhotsk Sea-West Pacific Stock of Minke Whales. Paper SC/43/Mi32 Presented to the IWC Scientific Committee, May 1991, 17 p. (Unpublished)

[8] Goto, M. and Pastene, L.A. (1997) Population Structure of Western North Pacific Minke Whale Based on an RFLP Analysis of MtDNA Control Region. Reports of the International Whaling Commission, 47, 531-537.

[9] Kanda, N., Goto, M., Kishiro, T., Yoshida, H., Kato, H. and Pastene, L.A. (2009) Individual Identification and Mixing of the J and O Stocks around Japanese Waters Examined by Microsatellite Analysis. Paper SC/J09/JR26 Presented to the JARPN II Review Workshop, Tokyo, January 2009, 9 p. (Unpublished)

[10] Kato, H. (1992) Body Length, Reproduction and Stock Separation of Minke Whales off Northern Japan. Reports of the International Whaling Commission, 42, 443-453.

[11] Bando, T., Kanda, N., Pastene, L.A., Kishiro, T., Yoshida, H. and Hatanaka, H. (2010) An Analysis of Cookie Cutter Shark-Induced Body Scar Marks of Common Minke Whales Sampled by JARPNII in the Context of Stock Structure Hypotheses. Paper SC/D10/NPM6 Presented to the First Intersessional Workshop of Western North Pacific Minke whale Implementation, December 2010, 5 p. (Unpublished)

[12] Hakamada, T. and Bando, T. (2009) Morphometric Analysis on Stock Structure in the Western North Pacific Common Minke Whales (Balaenoptera acutorostrata). Paper SC/J09/JR27. Presented to the JARPN II Review Workshop, Tokyo, January 2009, 13 p. (Unpublished)

[13] Kanda, N., Goto, M., Nagatsuka, S., Kato, H., Pastene, L.A. and Hatanaka, H. (2010) Analysis of Genetic and Non-Genetic Data Do Not Support the Hypothesis of an Intermediate Stock in Sub-Area 7. SC/D10/NPM8 Presented to the First Intersessional Workshop of Western North Pacific Minke Whale Implementation, December 2010, 8 p. (Unpublished) 
[14] Nagatsuka, S. (2010) External Morphological Difference of the Common Minke Whale (Balaenoptera acutorostrata) in the Western North Pacific. Master's Thesis, Tokyo University of Marine Science and Technology, Tokyo. (In Japanese) 